\title{
Derivation of transfer function from relay feedback systems
}

Ren Chiou Chang, Shih Haur Shen, and Cheng Ching Yu

Ind. Eng. Chem. Res., 1992, 31 (3), 855-860 • DOI: 10.1021/ie00003a030

Downloaded from http://pubs.acs.org on November 28, 2008

\section{More About This Article}

The permalink http://dx.doi.org/10.1021/ie00003a030 provides access to:

- Links to articles and content related to this article

- Copyright permission to reproduce figures and/or text from this article 
Chem. Process Des. Dev. 1983, 22 (3), 482-487.

Ramirez, W. F. Optimal State and Parameter Identification. An Application to Batch Beer Fermentation. Chem. Eng. Sci. 1987, $42,2749-2756$.

Shannon, R. E. Systems Simulation: The Art and Science; Prentice-Hall: Englewood Cliffs, NJ, 1975; p 371.

Stephanopoulos, G.; San, K. Y. Studies on On-line Bioreactor Identification: Theory. Biotechnol. Bioeng. 1984, 26, 1176-1188.

Tappi Standards and Suggested Methods; Technical Association of the Pulp and Paper Industry: 360 Lexington Avenue, New York, 1962.
Venkateswarlu, Ch.; Gangiah, K.; Jatkar, D. D.; Sridharan, S. S. Modelling and Simulation of Kraft Pulping Digester. Indian J. Technol. 1986, 24, 667-670.

Vroom, K. E. The H-factor: A Means of Expressing Cooking Times and Temperatures as a Single Variable. Pulp Pap. Mag. Can. $1957,58,228-231$.

Wells, C. H. Application of Modern Estimation and Identification Techniques to Chemical Processes. AIChE J. 1971, 17, 966-973.

Received for review September 9, 1991 Accepted October 30, 1991

\title{
Derivation of Transfer Function from Relay Feedback Systems
}

\author{
Ren-Chiou Chang, Shih-Haur Shen, and Cheng-Ching Y $\mathbf{u}^{*}$ \\ Department of Chemical Engineering, National Taiwan Institute of Technology, Taipei, Taiwan 10772, ROC
}

Luyben proposed a procedure for system identification based on the relay feedback system. This approach can lead to significant errors in the system parameters for transfer functions with time delay. Analytical expressions for the period of oscillation and amplitude ratios are derived. On the basis of these expressions, a procedure is proposed for deriving transfer functions. The results show that improvement can be achieved using the proposed method.

\section{Introduction}

On the basis of the Aström-Hägglund (1984) relay feedback system (ATV, autotune variation), Luyben (1987) proposed a procedure for the identification of process transfer functions. The procedure consists of two steps. First, a relay feedback experiment is conducted, and the ultimate gain and ultimate frequency are recorded. Second, this information is fitted to a typical transfer function in chemical process control, e.g., first-, second- or thirdorder plus time delay system. This identification approach, called the original ATV method hereafter, becomes a standard practice in chemical process control, as can be seen in recent textbooks in process control (Seborg et al., 1989, p 301; Luyben, 1990, p 520). The distinct advantages of the ATV method are as follows: (1) it identifies process information around the important frequency, ultimate frequency (the frequency where the phase angle is $-\pi$ ); (2) it is a closed-loop test, and therefore the process will not drift away from the nominal operating point; and (3) it is a more efficient method than conventional step or pulse testing (requires less time in the experiment). Despite the apparent success in industrial applications, the ATV method can be improved significantly to obtain a more accurate estimate of the system parameters. The reason is that the estimated ultimate gain $\hat{K}_{\mathrm{u}}$ and estimated ultimate frequency $\hat{\omega}_{u}$ derived from the describing function are only an approximation of information at the critical frequency. This approximation can lead to significant errors in the ultimate gain $K_{\mathrm{u}}$ and ultimate frequency $\omega_{\mathrm{u}}$ (e.g., 5-20\% error in $K_{u}$ ) for typical transfer functions in process control systems.

The purpose of this work is to derive analytical expressions for the period of oscillation and amplitude ratio for the relay feedback systems such that a better estimation of the process transfer function can be achieved. This paper is organized as follows. Section 2 reviews the ATV method of Luyben (1987). Analytical expressions for the period of oscillation and amplitude ratio are derived for typical process transfer functions (models 1-5 of Luyben (1987)), and an identification procedure based on the new results is presented in section 3. Examples are used to illustrate the accuracy of the proposed procedure, and the

\footnotetext{
* To whom all correspondence should be addressed.
}

robustness with respect to errors in the time delay is investigated in section 4 , followed by Conclusions.

\section{Review of ATV Method}

The Àström-Hägglund relay feedback system is based on the observation that a feedback system in which the output lags behind the input by $-\pi$ rad may oscillate with a period $P_{\mathrm{u}}$. Figure 1 illustrates the relay feedback system. A relay of magnitude $h$ is inserted in the feedback loop. Initially, the input $u$ is increased by $h$. As the output $y$ starts to increase (after a time delay $D$ ), the relay decreases the input to a position $h$ below the steady-state value. Since the phase lag is $-\pi$, a limit cycle with a period $\hat{P}_{\mathrm{u}}$ results (Figure 1). The period of the limit cycle is the ultimate period. Therefore, the estimated ultimate frequency from this relay feedback experiment is

$$
\hat{\omega}_{\mathrm{u}}=2 \pi / \hat{P}_{\mathrm{u}}
$$

From the Fourier series expansion, the amplitude $a$ can be considered as the result of the primary harmonic of the relay output. Therefore, the ultimate gain can be approximated as (Ogata, 1970; Äström and Hägglund, 1984)

$$
\hat{K}_{\mathrm{u}}=4 h / \pi a
$$

After the relay feedback experiment, the estimated ultimate gain $\left(\hat{K}_{u}\right)$, estimated ultimate frequency $\left(\hat{\omega}_{u}\right)$, and the observed time delay are used to back-calculate the process transfer function. Typical transfer functions in process control are assumed and parameters are calculated. The transfer functions have the following forms (Luyben, 1987):

models 1-3

$$
G(s)=\frac{K_{\mathrm{p}} e^{-D s}}{(\tau s+1)^{n}} \quad n=1,2,3
$$

model 4

$$
G(s)=\frac{K_{\mathrm{p}} e^{-D s}}{\left(\tau_{1} s+1\right)\left(\tau_{2} s+1\right)}
$$

model 5

$$
G(s)=\frac{K_{\mathrm{p}} e^{-D s}}{\left(\tau_{1} s+1\right)^{2}\left(\tau_{2} s+1\right)}
$$



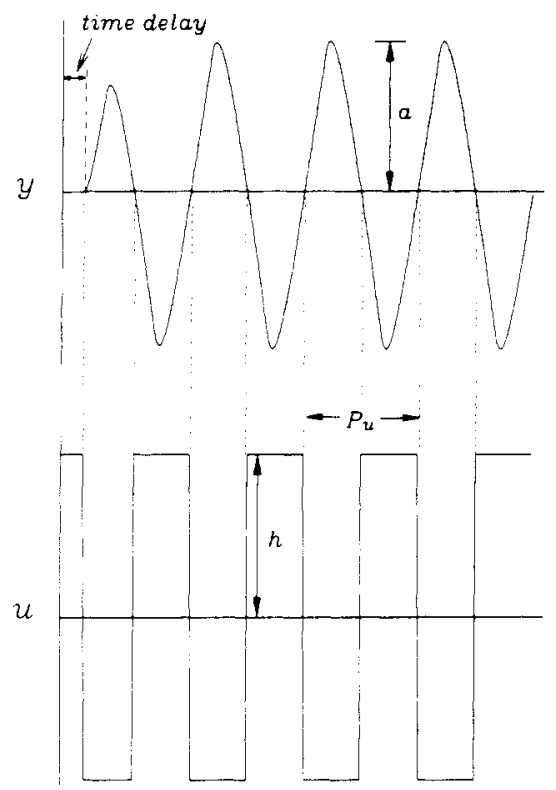

Figure 1. Relay feedback experiment.

The ATV method has the following steps (Luyben, 1987):

(1) The steady-state gain is obtained from steady-state simulation.

(2) Under relay feedback, the time delay $D$ is read off from the initial response (Figure 1 ).

(3) The ultimate gain $\left(\hat{K}_{u}\right)$ and ultimate frequency $\left(\hat{\omega}_{u}\right)$ are computed (eqs 1 and 2 ) after the relay feedback experiment.

(4) First-, second-, and third-order transfer functions (eqs 3-5) are fitted to the data at zero and the ultimate frequencies.

In theory, if the true ultimate gain and ultimate frequency are correctly identified, we can accurately find the parameters of a transfer function. For example, for a second-order with time delay system (eq 4), with $K_{\mathrm{p}}$ and $D$ available, we can back-calculate $\tau_{1}$ and $\tau_{2}$ from $\hat{K}_{\mathrm{u}}^{\mathrm{p}}$ and $\hat{\omega}_{\mathrm{u}}$ according to the following equations.

$$
\begin{gathered}
\frac{1}{\hat{K}_{\mathrm{u}}}=\frac{K_{\mathrm{p}}}{\left\{\left[1+\left(\hat{\omega}_{\mathrm{u}} \tau_{1}\right)^{2}\right]\left[1+\left(\hat{\omega}_{\mathrm{u}} \tau_{2}\right)^{2}\right]\right\}^{1 / 2}} \\
-\pi=-\hat{\omega}_{\mathrm{u}} D-\tan ^{-1}\left(\hat{\omega}_{\mathrm{u}} \tau_{1}\right)-\tan ^{-1}\left(\hat{\omega}_{\mathrm{u}} \tau_{2}\right)
\end{gathered}
$$

Unfortunately, since eqs 1 and 2 only give approximations to the ultimate gain and ultimate frequency, the parameters derived from eqs 6 and 7 can deviate significantly from the true system parameters. This implies that, for a linear second-order process (eq 4), the ultimate period is different from $\hat{P}_{u}$ observed from the relay feedback experiment (Figure 1) and the ultimate gain is not the same as the one derived in eq 2 .

\section{Analysis of Relay Feedback Systems}

In order to have a better approximation of the transfer function, fundamental analysis of the relay feedback system is necessary.

Period of Oscillation. One would like to know what the period of oscillation from the relay feedback experiment really represents. In other words, given a transfer function with known parameters, what is the expression for the period of oscillation observed from the relay feedback experiment, $\hat{P}_{\mathrm{u}}$. The following theorem (Aström and Hägglund, 1984) provides the answer.

Theorem (Astörm and Hägglund, 1984). Consider the relay feedback system with a transfer function $G(s)$ and an ideal relay (Figure 1 ). $\mathrm{HG}\left(T_{\mathrm{g}}, z\right)$ is given as the pulse transfer function of $G(s)$ with a sampling time of $T_{\mathrm{s}}$. If there is a periodic oscillation, then the period of oscillation $\hat{P}_{\mathrm{u}}$ is given by

$$
\mathrm{HG}\left(\hat{P}_{\mathrm{u}} / 2,-1\right)=0
$$

Ästörm and Hägglund (1984) prove the theorem starting from the discrete-time state space equations. The result, $\mathrm{HG}\left(\hat{P}_{\mathrm{u}} / 2,-1\right)=0$, is obtained by finding the $z$-domain equivalent. The continuous-time response of an ideal relay (Figure 1) can be discretized at the point when the relay switches. The $z$-transforms of the input and output are $h /(z+1)$ and 0 , respectively. Since this is a self-oscillation system, the propagation of the input is described by the gain $\mathrm{HG}\left(\hat{P}_{\mathrm{u}} / 2,-1\right)$. This equation can be used to find the period of oscillation for a known system. In identification, $\hat{P}_{u}$ is observed from the response, and one is able to use this to back-calculate the system parameters. Unlike the continuous-time analysis based on the primary harmonic, the discrete-time expression gives a sound basis for finding the system parameters, since no assumption is made in the derivation.

On the basis of this theorem, a better relationship between $\hat{\omega}_{\mathrm{u}}$ (or $\hat{P}_{\mathrm{u}}$ ) and system parameters can be derived. For the transfer functions of interest (eqs 3-5), the following results can be derived from the modified $z$-transform (see Appendix for the derivation of model 4).

model 1 (first-order lag with time delay)

$$
\tau=\frac{\pi}{\hat{\omega}_{\mathrm{u}} \ln |2 \exp (D / \tau)-1|}
$$

model 2 (two equal first-order lags with time delay)

$$
\begin{aligned}
& \tau=2 \pi\left[m+(m-1) \exp \left(-\frac{\pi}{\tau \hat{\omega}_{\mathrm{u}}}\right)\right] / \hat{\omega}_{\mathrm{u}}[1+ \\
& \left.\exp \left(-\frac{\pi}{\tau \hat{\omega}_{\mathrm{u}}}\right)\right]\left[\exp \left(\frac{m \pi}{\tau \hat{\omega}_{\mathrm{u}}}\right)\left(1+\exp \left(-\frac{\pi}{\tau \hat{\omega}_{\mathrm{u}}}\right)\right)-2\right]
\end{aligned}
$$

where

$$
m=1-\frac{D \hat{\omega}_{\mathrm{u}}}{\pi}
$$

model 3 (three equal first-order lags with time delay)

$$
\begin{gathered}
\exp (a m T)(1+\exp (-a T))^{3}-2(1+\exp (-a T))^{2}+ \\
2 a T[\exp (-a T)-m(1+\exp (-a T)](1+\exp (-a T))- \\
a^{2} T^{2}\left[m^{2}+\left(m^{2}-1\right) \exp (-a T)+\right. \\
\left.\exp (-a T)\left[(m-1)^{2}(1+\exp (-a T))-1\right]\right]=0
\end{gathered}
$$

where

$$
\begin{gathered}
m=1-\frac{D \hat{\omega}_{\mathrm{u}}}{\pi} \\
T=\pi / \hat{\omega}_{\mathrm{u}} \\
a=1 / \tau
\end{gathered}
$$

model 4 (two unequal first-order lags with time delay)

$$
\begin{gathered}
\tau_{1}\left[\left(2 \exp \left(-\frac{m \pi}{\tau_{1} \hat{\omega}_{\mathrm{u}}}\right)\right) /\left(1+\exp \left(-\frac{\pi}{\tau_{1} \hat{\omega}_{\mathrm{u}}}\right)\right)\right]-\tau_{1}= \\
\tau_{2}\left[\left(2 \exp \left(-\frac{m \pi}{\tau_{2} \hat{\omega}_{\mathrm{u}}}\right)\right) /\left(1+\exp \left(-\frac{\pi}{\tau_{2} \hat{\omega}_{\mathrm{u}}}\right)\right)\right]-\tau_{2}
\end{gathered}
$$

where 


$$
m=1-\frac{D \hat{\omega}_{u}}{\pi}
$$

model 5 (two equal first-order lags and one first-order lag with time delay)

$$
\begin{aligned}
& \text { where } \\
& \qquad \begin{aligned}
m & =1-\frac{D \hat{\omega}_{\mathrm{u}}}{\pi} \\
T & =\pi / \hat{\omega}_{\mathrm{u}} \\
a & =1 / \tau_{1} \\
b & =1 / \tau_{2}
\end{aligned}
\end{aligned}
$$$$
\left[-(a-b)^{2}(1+\exp (-a T))^{2}(1+\exp (-b T)) / 2\right]+
$$$$
a b T(a-b) \exp (-a m T)[(1-m) \exp (-a T)-m](1+
$$$$
\exp (-b T))-b(2 a-b)(1+\exp (-a T))(1+\exp (-b T)) \times
$$$$
\exp (-a m T)+a^{2}(1+\exp (-a T))^{2} \exp (-b m T)=0
$$

Equations 8-12 show that for a given transfer function, $\hat{\omega}_{u}$ observed from a relay feedback experiment does not necessarily correspond to the frequency where the phase angle is $-\pi$. For example, in the ATV method (Luyben, 1987), for model 1 , the following equation is employed to find the system parameter $\tau$.

$$
-\pi=-\hat{\omega}_{u} D-\tan ^{-1}\left(\hat{\omega}_{u} \tau\right)
$$

Equation 13 differs substantially from eq 8, especially from systems with a small time delay; e.g., $D$ is small. Therefore, the expressions derived here give a better approximation of $G(s)$.

Amplitude Ratio. The next step is to derive a better expression for the amplitude ratio at the ultimate frequency, since the expression in eq 3 is only an approximation of the ultimate gain based on the first harmonic of the relay feedback system, which can be expressed in a Fourier series:

$$
u(t)=\frac{4 h}{\pi} \sum_{n=1}^{\infty} \frac{\sin ((2 n+1) \omega t)}{2 n+1}
$$

Taking a Laplace transform of the input, we have

$$
U(s)=\frac{4 h \omega}{\pi} \sum_{n=1}^{\infty} \frac{1}{s^{2}+[(2 n+1) \omega]^{2}}
$$

If the process $G(s)$ is assumed to be three unequal firstorder lags with time delay $D$

$$
G(s)=\frac{K_{\mathrm{p}} e^{-D_{s}}}{\left(\tau_{1} s+1\right)\left(\tau_{2} s+1\right)\left(\tau_{3} s+1\right)}
$$

then the output $Y(s)$ becomes

$$
\begin{aligned}
& Y(s)= \\
& \frac{4 h K_{\mathrm{p}} \omega}{\pi} \sum_{n=1}^{\infty} \frac{\exp (-D s)}{\left[s^{2}+((2 n+1) \omega)^{2}\right]\left(\tau_{1} s+1\right)\left(\tau_{2} s+1\right)\left(\tau_{3} s+1\right)}
\end{aligned}
$$

With the inverse Laplace transform, the output becomes

$$
\begin{aligned}
& y(t)=\frac{4 h K_{\mathrm{p}}}{\pi} \sum_{n=1}^{\infty}\{ {[\sin [(2 n+1) \omega(t-D)+} \\
&\left.\phi_{2 n+1}\right] /(2 n+1)\left[\left[1+\left((2 n+1) \tau_{1} \omega\right)^{2}\right][1+((2 n+\right. \\
&\left.\left.\left.\left.1) \tau_{2} \omega\right)^{2}\right]\left[1+\left((2 n+1) \tau_{3} \omega\right)^{2}\right]\right]^{1 / 2}\right]+c \exp \left(-\frac{t-D}{\tau_{1}}\right)+ \\
&\left.d \exp \left(-\frac{t-D}{\tau_{2}}\right)+f \exp \left(-\frac{t-D}{\tau_{3}}\right)\right\}
\end{aligned}
$$

where the constants $c, d$, and $f$ are functions of $n, \omega, \tau_{1}$, and $\tau_{2}$ and $\phi_{2 n+1}$ is the phase shift at $(2 n+1) \omega$. Under the self-oscillation condition, the output waveform (Figure 1) becomes

$$
\begin{aligned}
& y(t)=\frac{4 h K_{\mathrm{p}}}{\pi} \sum_{n=1}^{\infty} \sin \left[(2 n+1) \omega(t-D)+\phi_{2 n+1}\right] / \\
& (2 n+1)\left\{\left[1+\left((2 n+1) \tau_{1} \omega\right)^{2}\right]\left[1+\left((2 n+1) \tau_{2} \omega\right)^{2}\right] \times\right. \\
& \left.\left[1+\left((2 n+1) \tau_{3} \omega\right)^{2}\right]\right\}^{1 / 2}(19)
\end{aligned}
$$

Therefore, it becomes obvious that the amplitude observed in the relay feedback response is the contribution from multiple frequencies, $\omega=\hat{\omega}, 3 \hat{\omega}, 5 \hat{\omega}, \ldots$. For a strictly proper system, the contribution from higher frequencies, e.g., $3 \hat{\omega}$, is significantly less then that from the primary harmonic, as shown in eq 19. If only one term is employed, we have

$$
\frac{\pi a}{4 h}=\frac{K_{\mathrm{p}}}{\left\{\left[1+\left(\tau_{1} \omega\right)^{2}\right]\left[1+\left(\tau_{2} \omega\right)^{2}\right]\left[1+\left(\tau_{3} \omega\right)^{2}\right]\right\}^{1 / 2}}
$$

This is exactly the formula employed in the ATV method in finding the system parameters (Luyben, 1987), since $\hat{K}_{u}$ $=\pi a / 4 h$. In this work, eq 20 is employed in finding the system parameters. Specifically, we have

model 1

$$
\frac{\pi a}{4 h}=\frac{K_{\mathrm{p}}}{\left[1+\left(\tau \hat{\omega}_{\mathrm{u}}\right)^{2}\right]^{1 / 2}}
$$

model 2

$$
\frac{\pi a}{4 h}=\frac{K_{\mathrm{p}}}{1+\left(\tau \hat{\omega}_{\mathrm{u}}\right)^{2}}
$$

model 3

$$
\frac{\pi a}{4 h}=\frac{K_{\mathrm{p}}}{\left[1+\left(\tau \hat{\omega}_{\mathrm{u}}\right)^{2}\right]^{2 / 3}}
$$

model 4

$$
\frac{\pi a}{4 h}=\frac{K_{\mathrm{p}}}{\left\{\left[1+\left(\tau_{1} \hat{\mathrm{\omega}}_{\mathrm{u}}\right)^{2}\right]\left[1+\left(\tau_{2} \hat{\omega}_{\mathrm{u}}\right)^{2}\right]\right\}^{1 / 2}}
$$

model 5

$$
\frac{\pi a}{4 h}=\frac{K_{\mathrm{p}}}{\left[1+\left(\tau_{1} \hat{\omega}_{\mathrm{u}}\right)^{2}\right]\left[1+\left(\tau_{2} \hat{\omega}_{\mathrm{u}}\right)^{2}\right]^{1 / 2}}
$$

Identification Procedure. From the ongoing analysis, the procedure for the evaluation of the transfer function has the following steps: (1) same as (1) of the ATV method; (2) same as (2) of the ATV method; (3) $\hat{\omega}_{u}$ and $\pi a / 4 h$ (Figure 1) computed from the relay feedback experiment; (4) model 1-5 fitted to the data by solving the corresponding equations (eqs 8 and/or 21 for model 1, eqs 9 and/or 22 for model 2, eqs 10 and/or 23 for model 3, eqs 11 and 24 for model 4, and eqs 12 and 25 for model 5) for the unknown parameters.

In models 1-3, the time constant $\tau$ can be evaluated from two different equations and the difference between these $\tau$ 's is an indication of the appropriateness of the model structure. Generally, the low-order model that fits the data is used (Luyben, 1987). Notice that this is only one way to evaluate transfer functions. If some information is not known, then a different step should be employed. For example, if $K_{\mathrm{p}}$ is not available, models $1-3$ still can be evaluated, but models 4 and 5 require additional information (e.g., information at different frequencies; $\mathrm{Li}$ et al., 1991). Nonetheless, eqs 8-12 and 21-25 are generally 


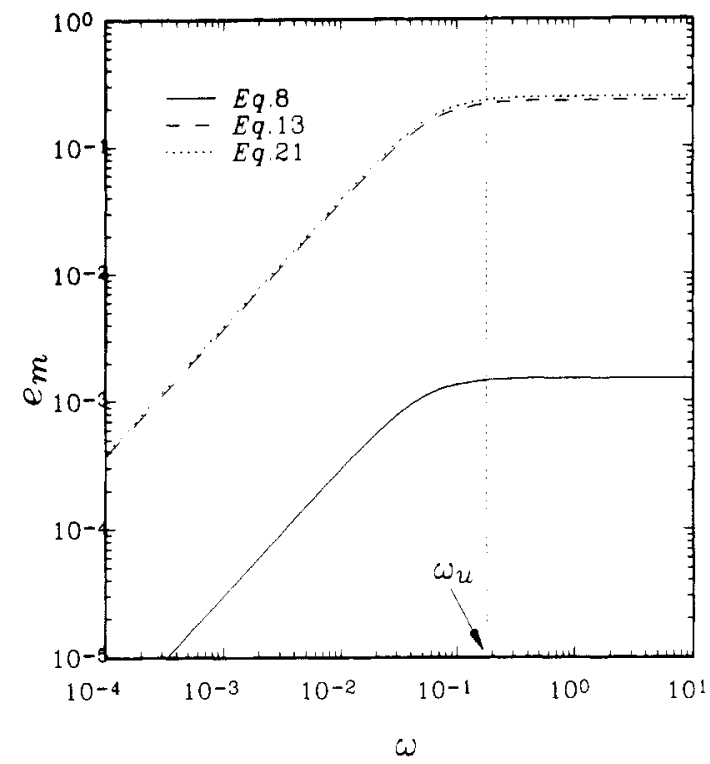

Figure 2. Multiplicative errors of first-order plus dead time system obtained from different equations (eqs 8, 13, 21).

applicable regardless of the procedure.

Theoretically, one can have a better estimate of the amplitude ratio by employing more terms according to eq 19. An iterative procedure is necessary if more than one term is employed (e.g., finding $G(s)$ from the single-term solution and including the higher frequency information, $\omega=3 \hat{\omega}_{u}$, to find a new $G(s)$, repeating the procedure until $G(s)$ converges). However, experimental results show that the estimation of system parameters can be improved substantially by improving the expression for the period of oscillation alone, as shown in the next section. Furthermore, for higher order systems, there is little incentive to improve the expression for the amplitude by including more terms in eq 19 since higher order harmonics (e.g., $\omega$ $=3 \hat{\omega}_{\mathrm{u}}$ or $\omega=5 \hat{\omega}_{\mathrm{u}}$ ) contribute little to the amplitude.

\section{Results and Discussions}

Several examples are used to illustrate the advantages of the proposed procedure. Consider a first-order system with time delay

$$
G(s)=\frac{1.65 e^{-10 s}}{20 s+1}
$$

From a relay feedback experiment with $h=0.04$, we have $\hat{P}_{\mathrm{u}}=33.26$ and $a=0.26$. On the basis of this information we can back-calculate $\tau$. The $\tau$ 's calculated from eqs 13 and 21 , the original ATV method, are $\tau=16.3$ and 16.086, respectively. The proposed method gives a better estimate in $\tau, \tau=19.97$, by improving the expression in the time period of oscillation alone (eq 8). The result from eq 8 is almost the exact solution (the difference may result from reading off $a$ and $P_{\mathrm{u}}$ from the response curve). Figure 2 shows the multiplicative modeling errors, $e_{\mathrm{m}}=\mid(G(i \omega)-$ $\hat{G}(i \omega)) / \hat{G}(i \omega) \mid$, for the transfer function $\hat{G}$ estimated from eqs 8,13 , and 21 . The results show that the error, $e_{m}$, is significant less when $\tau$ is calculated from eq 8 alone. Therefore, in this work, the expressions for the period of oscillation (eqs 8-10) are employed for identifying the system parameter for models 1-3. For the original ATV method, the time constant $\tau$ for models $1-3$ is obtained by taking the average of the values calculated from the corresponding pair of equations (e.g., eqs 8 and 13 for model 1).

Next the effects of time delay on the estimation of ultimate gain and ultimate frequency are also investigated.
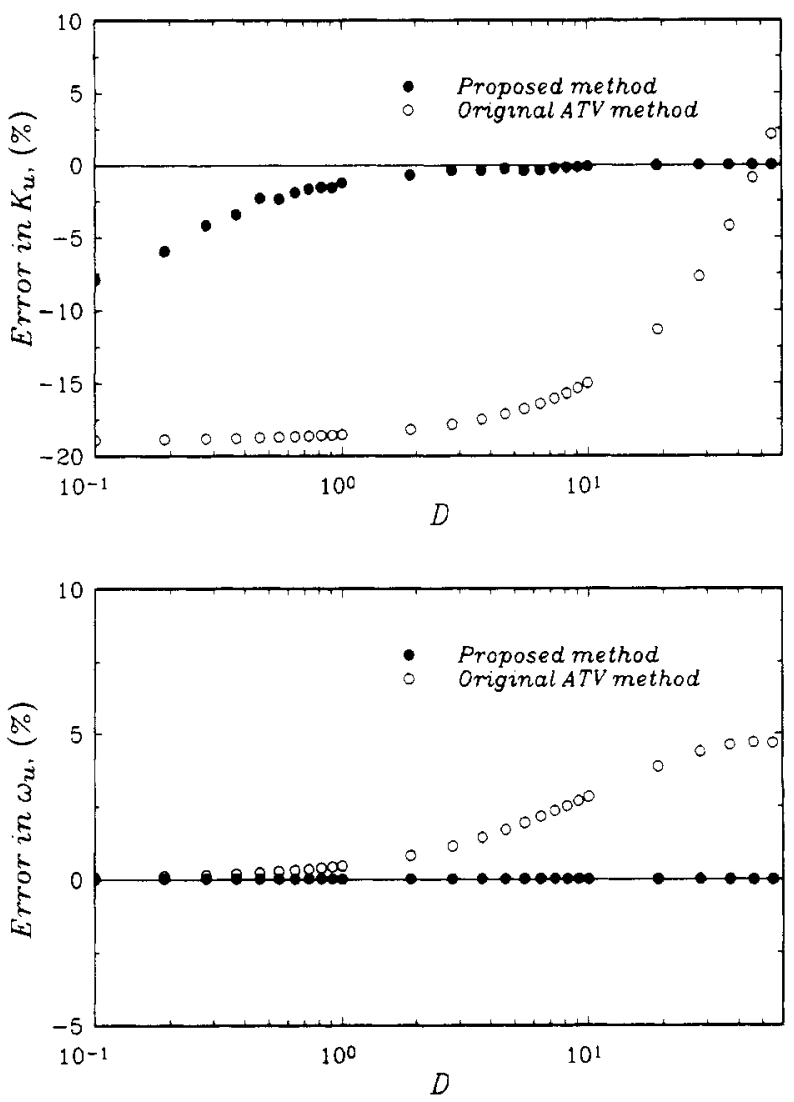

Figure 3. Percent errors in $K_{u}$ and $\omega_{u}$ for the first-order lag over a range of time delay $D$.

In the original ATV method $\hat{K}_{\mathrm{u}}$ is calculated from eq 2 and $\hat{\omega}_{u}$ is derived from eq 1 . In the proposed method, $K_{u}$ and $\omega_{u}$ are back-calculated from the estimated transfer function $\hat{G}(s)$. Again, for the following transfer function

$$
G(s)=\frac{1.65 e^{-D s}}{20 s+1}
$$

The percent errors in $K_{\mathrm{u}}$ and $\omega_{\mathrm{u}}$ are compared for these two methods over a range of time delay $(D=0.1-60)$. The results (Figure 3) show that the errors in $K_{\mathrm{u}}$ for the original ATV method are quite significant (5-20\%). Furthermore, the error in $\omega_{\mathrm{u}}$ is almost nil for the proposed method. The proposed method improves the estimation significantly. Similar behavior can also be observed for a second-order lag with time delay system.

$$
G(s)=\frac{37.3 e^{-D s}}{(7200 s+1)(2 s+1)}
$$

Figure 4 shows a better estimation of $\hat{G}(s)$ can be achieved over a range of $D(D<10)$. Again, improvements can be made in finding the correct $K_{\mathrm{u}}$ and $\omega_{\mathrm{u}}$ by using a more accurate expression in the period of oscillation.

Since the estimated transfer function is typically employed in the analysis and design of the feedback control system, the "impact" of the modeling errors in closed-loop performance is evaluated. A model-based controller, IMC (internal model control), is employed to analyze the performance. One of the advantages of the model-based controllers, e.g., IMC, is that we can specify the desired trajectory in the design. Figure 5 compares the set point responses of IMC when different models, $\hat{G}$ 's, are employed in the design of the controllers. For the first-order plus time delay system $G(s)=1.65 e^{-10 s} /(20 s+1)$, the set point response of the control system, designed according to $\hat{G}(s)$ from the original ATV method, tends to be more sluggish 

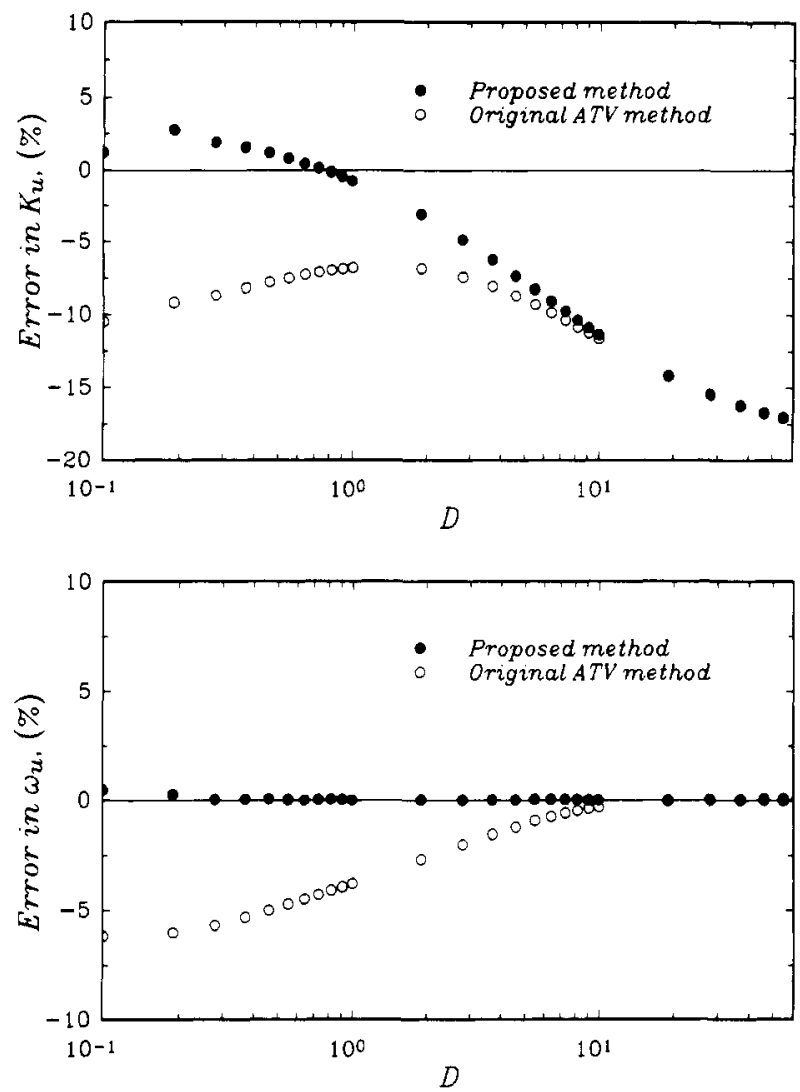

Figure 4. Percent errors in $K_{u}$ and $\omega_{u}$ for two unequal first-order lags with time delay systems over a range of delay time $D$.

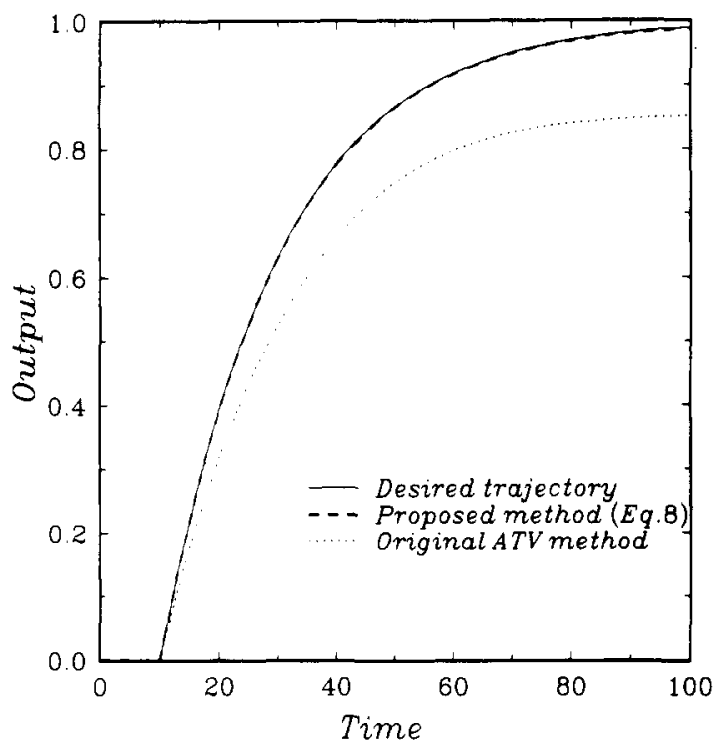

Figure 5. Set point responses of IMC designed according to the estimated transfer function $\hat{G}(s)$ obtained from the proposed method and the original ATV method (the closed-loop time constant is 20 for the desired trajectory).

than the desired trajectory (Figure 5). The proposed method improves this situation, as shown in Figure 5. Despite the fact that a tighter response can be achieved by shortening the closed-loop time constant under modeling errors, one has to realize that the value of a modelbased controller is that one can foresee the closed-loop response. In other words, a good model always helps.

Generally, the proposed method improves the estimation in $G(s)$ at the nominal condition (with perfect knowledge in $K_{\mathrm{p}}$ and $D$ ). The robustness of the proposed method with respect to errors in the delay is investigated. Since the
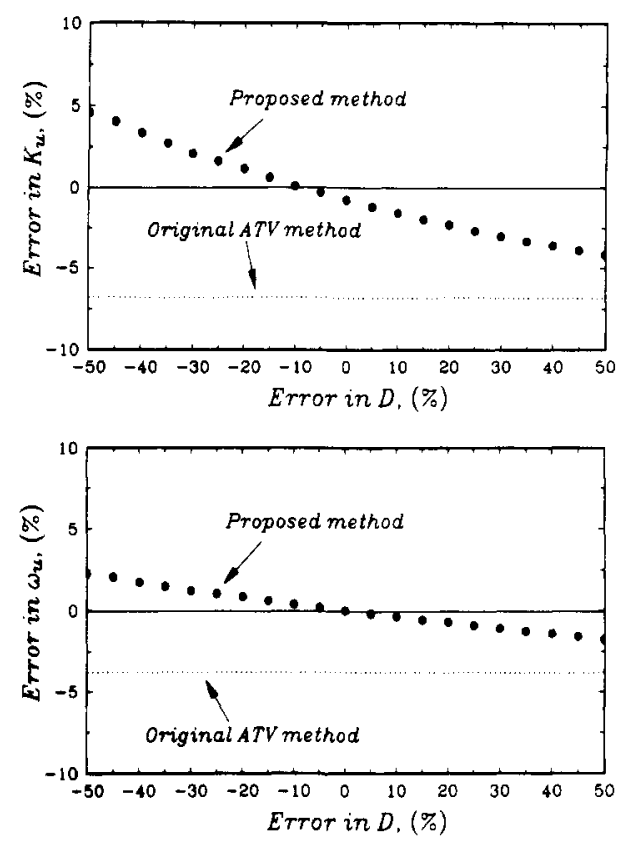

Figure 6. Percent errors in $K_{\mathrm{u}}$ and $\omega_{\mathrm{u}}$ for two unequal first-order lags over a range of variation in the time delay errors.

proposed method calculates $K_{\mathrm{u}}$ and $\omega_{\mathrm{u}}$ by finding the transfer function $\hat{G}(s)$ first followed by solving the corresponding equations for them, it is more sensitive to the errors in the time delay than the original ATV method (ATV method finds $\hat{K}_{\mathrm{u}}$ and $\hat{\omega}_{\mathrm{u}}$ from the response directly provided with eqs 1 and 2). Another first-order plus time delay system is given as an example.

$$
G(s)=\frac{1.65 e^{-s}}{20 s+1}
$$

Figure 6 shows the estimate of $K_{\mathrm{u}}$ and $\omega_{\mathrm{u}}$ for both methods when the percent errors in time delay ranges from -50 to $50 \%$. Despite the fact that the errors in $K_{\mathrm{u}}$ and $\omega_{\mathrm{u}}$ are less for the proposed method over the reasonable range of errors in time delay, it is more sensitive to errors, as shown in Figure 6. Therefore, precaution should be taken in reading off the time delay from the initial responses.

\section{Conclusions}

Recently, system identification based on the relay feedback system, the ATV method, has become a standard practice in process control. However, this approach can lead to error if the system parameters are estimated from the approximated ultimate gain $\left(\hat{K}_{\mathrm{u}}\right.$, eq 2$)$ and ultimate frequency $\left(\hat{\omega}_{u}\right.$, eq 1$)$. Analytical expressions are derived for the period of oscillation (eqs 8-12) and amplitude ratio (eq 19). On the basis of these results, an identification procedure is proposed. The results show that the proposed method results in a better estimate of the process transfer function. Precaution should be taken in reading off the time delay, since the proposed method is more sensitive to errors in time delay than the original ATV method.

\section{Acknowledgment}

This work is supported by the National Science Council of the ROC under Grant No. NSC 81-0402-E-011-07.

\section{Nomenclature}

$a=$ amplitude of limit cycle

$D=$ time delay

$G(s)=$ nominal process transfer function 
$\hat{G}(s)=$ estimated process transfer function

$\mathrm{HG}\left(T_{s}, z\right)=$ pulse transfer function

$h=$ magnitude of relay output

$K_{\mathrm{p}}=$ steady-state gain

$K_{\mathrm{u}}=$ ultimate gain

$\hat{K}_{\mathrm{u}}=$ estimated ultimate gain

$P_{\mathrm{u}}=$ ultimate period

$\hat{P}_{\mathrm{u}}^{\mathrm{u}}=$ estimated ultimate period

$T_{\mathrm{B}}=$ sampling time

$u=$ input

$y=$ output

$z=z$-transform variable

Greek Symbols

$\tau=$ time constant

$\omega=$ frequency

$\omega_{u}=$ ultimate frequency

$\hat{\omega}_{\mathrm{u}}=$ estimated ultimate frequency

\section{Acronyms}

ATV $=$ autotune variation

\section{Appendix}

A two unequal first-order lag with time delay transfer function is considered

$$
G(s)=\frac{K_{\mathrm{p}} e^{-D_{s}}}{\left(\tau_{1} s+1\right)\left(\tau_{2} s+1\right)}
$$

Equation $\mathrm{A} 1$ can be decomposed into

$$
G(s)=\frac{K_{\mathrm{p}}}{\tau_{1}-\tau_{2}} e^{-D s}\left(\frac{\tau_{1}}{\tau_{1} s+1}-\frac{\tau_{2}}{\tau_{2} s+1}\right)
$$

The pulse transfer function of $G(s)$ is

$$
\mathrm{HG}\left(T_{\mathrm{s}}, z\right)=\frac{K_{\mathrm{p}}}{\tau_{1}-\tau_{2}}\left(\frac{a_{1} z^{-1}+a_{2} z^{-2}}{1+a_{3} z^{-1}} \tau_{1}-\frac{b_{1} z^{-1}+b_{2} z^{-2}}{1+b_{3} z^{-1}} \tau_{2}\right)
$$

where

$$
\begin{gathered}
a_{1}=1-\exp \left(\frac{D-T_{\mathrm{s}}}{\tau_{1}}\right) \\
a_{2}=\exp \left(-\frac{T_{\mathrm{s}}}{\tau_{1}}\right)\left(\exp \left(\frac{D}{\tau_{1}}\right)-1\right) \\
a_{3}=-\left(\exp \left(-\frac{T_{8}}{\tau_{1}}\right)\right) \\
b_{1}=1-\exp \left(\frac{D-T_{\mathrm{s}}}{\tau_{2}}\right)
\end{gathered}
$$

$$
\begin{gathered}
b_{2}=\exp \left(-\frac{T_{\mathrm{s}}}{\tau_{2}}\right)\left(\exp \left(\frac{D}{\tau_{2}}\right)-1\right) \\
b_{3}=-\left(\exp \left(-\frac{T_{\mathrm{s}}}{\tau_{2}}\right)\right)
\end{gathered}
$$

According to the theorem of Áström and Hägglund, we have

$$
\begin{aligned}
& \operatorname{HG}\left(\hat{P}_{\mathrm{u}} / 2,-1\right)=\left\{\left[-\left(1-\exp \left(\frac{D-\hat{P}_{\mathrm{u}} / 2}{\tau_{1}}\right)\right)+\exp \left(-\frac{\hat{P}_{\mathrm{u}}}{2 \tau_{1}}\right) \times\right.\right. \\
&\left.\left(\exp \left(\frac{D}{\tau_{1}}\right)-1\right) \tau_{1} /\left[1+\exp \left(-\frac{\hat{P}_{\mathrm{u}}}{2 \tau_{1}}\right)\right]\right\} \\
&\left\{\left[-\left(1-\exp \left(\frac{D-\hat{P}_{\mathrm{u}} / 2}{\tau_{2}}\right)\right)+\exp \left(-\frac{\hat{P}_{\mathrm{u}}}{2 \tau_{2}}\right) \times\right.\right. \\
&\left.\left.\left(\exp \left(\frac{D}{\tau_{2}}\right)-1\right)\right] \tau_{2} /\left[1+\exp \left(-\frac{\hat{P}_{\mathrm{u}}}{2 \tau_{2}}\right)\right]\right\} \\
& \mathrm{HG}\left(\hat{P}_{\mathrm{u}} / 2,-1\right)=0
\end{aligned}
$$

Rearranging eq A4, we get

$$
\begin{aligned}
& \tau_{1}\left[\left(2 \exp \left(-\frac{m \pi}{\tau_{1} \hat{\omega}_{\mathrm{u}}}\right)\right) /\left(1+\exp \left(-\frac{\pi}{\tau_{1} \hat{\omega}_{\mathrm{u}}}\right)\right)\right]-\tau_{1}= \\
& \tau_{2}\left[\left(2 \exp \left(-\frac{m \pi}{\tau_{2} \hat{\omega}_{\mathrm{u}}}\right)\right) /\left(1+\exp \left(-\frac{\pi}{\tau_{2} \hat{\omega}_{\mathrm{u}}}\right)\right)\right]-\tau_{2}
\end{aligned}
$$

Equations $8-10$ and 12 can be derived in a similar manner.

\section{Literature Cited}

Äström, K. J.; Hägglund, T. Automatic Tuning of Simple Regulators with Specifications on Phase and Amplitude Margins. Automatica 1984, 20,645-651.

Li, W.: Eskinat, E.; Luyben, W. L. An Improved Autotune Identification Method. Ind. Eng. Chem. Res. 1991, 30, 1530-1541.

Luyben, W. L. Derivation of Transfer Functions for Highly Nonlinear Distillation Columns. Ind. Eng. Chem. Res. 1987, 26, 2490-2495.

Luyben, W. L. Process Modeling, Simulation and Control for Chemical Engineers; McGraw-Hill: New York, 1990.

Ogata, K. Modern Control Engineering; Prentice Hall: Englewood Cliffs, NJ, 1970; Chapter 11.

Seborg, D. E.; Edgar, T. F.; Mellichamp, D. A. Process Dynamics and Control; Wiley: New York, 1989.

Received for review June 11, 1991 Revised manuscript received November 7, 1991 Accepted November 28, 1991 